\title{
Azithromycin is able to control Toxoplasma gondii infection in human villous explants
}

\author{
Letícia S Castro-Filice ${ }^{1}$, Bellisa F Barbosa', Mariana B Angeloni', Neide M Silva², Angelica O Gomes ${ }^{1}$, \\ Celene M O S Alves ${ }^{1}$, Deise A O Silva², Olindo A Martins-Filho ${ }^{3}$, Maria C Santos ${ }^{4}$, José R Mineo ${ }^{2}$ \\ and Eloisa A V Ferro ${ }^{1 *}$
}

\begin{abstract}
Background: Although Toxoplasma gondii infection is normally asymptomatic, severe cases of toxoplasmosis may occur in immunosuppressed patients or congenitally infected newborns. When a fetal infection is established, the recommended treatment is a combination of pyrimethamine, sulfadiazine and folinic acid (PSA). The aim of the present study was to evaluate the efficacy of azithromycin to control T. gondii infection in human villous explants.

Methods: Cultures of third trimester human villous explants were infected with T. gondii and simultaneously treated with either PSA or azithromycin. Proliferation of T. gondii, as well as production of cytokines and hormones by chorionic villous explants, was analyzed.

Results: Treatment with either azithromycin or PSA was able to control T. gondii infection in villous explants. After azithromycin or PSA treatment, TNF-a, IL-17A or TGF- $\beta 1$ levels secreted by infected villous explants did not present significant differences. However, PSA-treated villous explants had decreased levels of IL-10 and increased IL-12 levels, while treatment with azithromycin increased production of IL-6. Additionally, T. gondii-infected villous explants increased secretion of estradiol, progesterone and $\mathrm{HCG}+\beta$, while treatments with azithromycin or PSA reduced secretion of these hormones concurrently with decrease of parasite load.
\end{abstract}

Conclusions: In conclusion, these results suggest that azithromycin may be defined as an effective alternative drug to control T. gondii infection at the fetal-maternal interface.

Keywords: Azithromycin, Human placental villi, Trophoblast, Cytokines, Sex hormones, Toxoplasma gondii

\section{Introduction}

Toxoplasma gondii is an obligate intracellular protozoan parasite that infects a wide range of hosts, including humans. During pregnancy, primary infection can result in the vertical transmission of $T$. gondii tachyzoites, potentially having severe consequences for the fetus, such as retinochoroiditis as well as hydrocephalus and intracranial calcification [1]. The risk of fetal infection by $T$. gondii increases as pregnancy progresses [2,3]. Nevertheless, the consequences of fetal infection are more severe the earlier infection occurs during pregnancy [4].

\footnotetext{
* Correspondence: eloisa@umuarama.ufu.br

'Laboratory of Immunophysiology of Reproduction, Institute of Biomedical Sciences, Universidade Federal de Uberlândia, Av. Pará 1720, Uberlândia, MG 38400-902, Brazil

Full list of author information is available at the end of the article
}

A combination of pyrimethamine, sulfadiazine and folinic acid (PSA) or spiramycin are standard care for treatment of toxoplasmosis in cases of fetal infection [5,6]. Treatment with spiramycin is administered immediately after diagnosis of maternal infection [7], and since this macrolide does not cross placenta, it is not suitable for treatment of fetal infections [8]. PSA is the recommended combination for pregnant women treatment who acquire the infection after 18 weeks of gestation. However, pyrimethamine is potentially teratogenic and should not be used in the first trimester of pregnancy [8]. Pyrimethamine is a folic acid antagonist and its use in pregnancy has been associated with increased risk of neural tube defects [9]. This drug is potentially toxic, usually causing gradual dose-related bone marrow depression, chromosomal damage and mutagenicity $[9,10]$. Folinic acid is used for reduction and prevention of hematological toxicities caused by 
pyrimethamine [8]. Alternative drugs for treatment of toxoplasmosis, such as trimethoprim-sulfamethoxazole or clindamycin, have shown activity in vitro and in mouse models, but clinical studies to establish their efficacy have not been conclusive [10]. Thus, there is an urgent need for more effective and non-toxic chemotherapeutic agents and novel drug targets to be identified for treatment of $T$. gondii infection during pregnancy.

Azithromycin is a semisynthetic azalide antibiotic that is structurally related to erythromycin, but has a broader spectrum of antibacterial activity and a more favorable pharmacokinetic profile. Furthermore, administration is required only once per day [11-13]. Azithromycin also inhibits protein synthesis in the plasmodial apicoplast and, thus, has activity against both Plasmodium falciparum and $P$. vivax [12]. Azithromycin is widely used for the treatment of community-acquired pneumonia and chlamydia during pregnancy, and has been safely administered in all trimesters of gestation $[14,15]$. Studies have shown that animals receiving 60 times the recommended dose of azithromycin for humans do not have decreased fertility or demonstrated harmful effects on the fetus [16]. Given that azithromycin is considered safe during pregnancy and the fact that it may have activity against pathogens, such as Plasmodium and Toxoplasma, it has been suggested as a candidate for intermittent preventive treatment during pregnancy $[7,17]$. Previous studies have shown that azithromycin treatment in pregnant Calomys callosus and human trophoblast cells (lineage BeWo) was able to control $T$. gondii infection, suggesting that it may be an alternative drug for prevention of congenital infection $[18,19]$. Additionally, azithromycin has anti-inflammatory properties through modulating the production of proinflammatory cytokines that are produced during $T$. gondii infection [20].

Thus, the aim of the present study was to evaluate the efficacy of azithromycin in the control of T. gondii infection in third trimester human villous explants cultures compared to traditional therapy (PSA).

\section{Methods}

\section{Placental samples}

Placental tissues were obtained from 24 women after elective cesarean section deliveries (36 to 40 weeks of pregnancy). Exclusion criteria included pre-eclampsia, chronic hypertension, infectious disease including toxoplasmosis, chorioamnionitis, chronic renal disease, cardiac disease, connective tissue disease, pre-existing diabetes mellitus and gestational diabetes mellitus. Placental tissues were placed in ice-cold sterile phosphate-buffered saline (PBS), $\mathrm{pH} 7.2$, to remove excess blood then aseptically dissected using a stereomicroscope to remove endometrial tissue and fetal membranes up to $1 \mathrm{~h}$ after collection. Floating terminal chorionic villous explants containing five to seven free tips per explant were collected as described previously [21,22]. Explants were added to 96-well plates (one per well) and cultured in complete medium containing RPMI 1640 medium (Cultilab, Campinas, SP, Brazil) supplemented with $10 \%$ fetal bovine serum (FBS) (Cultilab), $100 \mathrm{U} / \mathrm{mL}$ penicillin, and $100 \mu \mathrm{g} / \mathrm{mL}$ streptomycin (Sigma-Aldrich Co., St. Louis, MO, USA) for $24 \mathrm{~h}$ at $37^{\circ} \mathrm{C}$ and $5 \% \mathrm{CO}_{2}$.

\section{Parasites}

Tachyzoites from $T$. gondii 2 F1 strain, which constitutively express cytoplasmic $\beta$-galactosidase and are derived from the RH strain, were maintained by serial passages in BeWo cells in RPMI medium supplemented with penicillin, streptomycin and $2 \% \mathrm{FBS}$ at $37^{\circ} \mathrm{C}$ and $5 \% \mathrm{CO}_{2}$. T. gondii 2 F1 was a gift from Dr. Vern Carruthers, Medicine School of Michigan University (USA).

\section{Antibiotics}

Macrolide azithromycin (Biofarma, Uberlândia, MG, Brazil) or a drug combination consisting of pyrimethamine (Daraprim ${ }^{\bullet}$ - Farmoquímica, Rio de Janeiro, RJ, Brazil), sulfadiazine (Suladrin ${ }^{\oplus}$ - Laboratório Catarinense, Joinville, SC, Brazil) and folinic acid (Genix, Anápolis, GO, Brazil) (PSA) were dissolved in culture medium to a concentration of $5000 \mu \mathrm{g} / \mathrm{mL}$ (stock solution). Different drug concentrations were prepared by diluting the stock solution and used for treatment of villous explants.

\section{Treatments}

First, villous explants were infected with $T$. gondii 2 F1 strain tachyzoites $\left(1 \times 10^{6}\right.$ parasites per well $)$ and incubated for 48 h. Next, villous explants were washed with complete medium to remove non-adhered parasites and treated for an additional $24 \mathrm{~h}$ with different concentrations of azithromycin (200, 1000 or $5000 \mu \mathrm{g} / \mathrm{mL})$ or PSA as follows: 200, 1000 or $5000 \mu \mathrm{g} / \mathrm{mL}$ for pyrimethamine; 150,750 or $3750 \mu \mathrm{g} / \mathrm{mL}$ for sulfadiazine; and 30, 150 or $750 \mu \mathrm{g} / \mathrm{mL}$ for folinic acid, as previously established [23]. Uninfected/untreated villous explants or infected/untreated villous explants (NT) were cultured with complete medium only and included as experimental controls. After, culture supernatants were collected and stored at $-70^{\circ} \mathrm{C}$ for measurement of cytokines or nitrite at a later time. Villous explants were collected for morphological analysis, immunohistochemistry, or analysis for $T$. gondii intracellular proliferation using a colorimetric $\beta$-galactosidase assay as previously described [24]. T. gondii intracellular proliferation data were expressed as the number of tachyzoites calculated in relation to the standard curve of 2 F1 strain tachyzoites ranging from $1 \times 10^{6}$ to $15.625 \times 10^{3}$ total parasites.

In another set of experiments, parasites were pre-treated with antibiotics. Briefly, 2 F1 strain tachyzoites were treated 
with different concentrations of azithromycin (50, 100, 200, $1000 \mu \mathrm{g} / \mathrm{mL})$ or PSA $(50,100,200$ or $1000 \mu \mathrm{g} / \mathrm{mL}$ for pyrimethamine; 37.5, 75, 150 or $750 \mu \mathrm{g} / \mathrm{mL}$ for sulfadiazine and $7.5,15,30$ or $150 \mu \mathrm{g} / \mathrm{mL}$ for folinic acid), diluted in culture complete medium for $1 \mathrm{~h}$ at $37^{\circ} \mathrm{C}$ in a $\mathrm{CO}_{2}$ incubator. Afterwards, parasites were placed in 96-well tissue culture plates containing previously collected villous explants. Untreated parasites (NTp) remained for $1 \mathrm{~h}$ in complete medium under the same conditions as treated parasites and were used to infect villous explants. After $72 \mathrm{~h}$, villous explants were collected for $T$. gondii proliferation assays according Teo et al. [24].

\section{T. gondii intracellular proliferation assay}

The villous explants were submitted to a colorimetric $\beta$-galactosidase assay in order to analyze the $T$. gondii intracellular proliferation [24] with minor modifications. Briefly, villous explants were homogenized in radioimmunoprecipitation assay buffer (RIPA) [50 mmol/L Tris hydrochloride, $150 \mathrm{mmol} / \mathrm{L} \mathrm{NaCl}, 1 \%$ (v/v) Triton $\mathrm{X}-100,1 \%(\mathrm{w} / \mathrm{v})$ sodium deoxycholate, and $0.1 \%(\mathrm{w} / \mathrm{v})$ SDS; pH 7.5] plus protease inhibitor cocktail tablets (Roche Diagnostics GmbH, Mannheim, Germany). The homogenate was centrifuged at 15,000 $\mathrm{x} g$ for 15 minutes at $4{ }^{\circ} \mathrm{C}$, the supernatants were used for protein content measurement using the Bradford method [25] and $100 \mu \mathrm{g} / \mathrm{mL}$ of total protein was used to perform the colorimetric $\beta$-galactosidase assay. Then, the total protein $(100 \mu \mathrm{g} / \mathrm{mL})$ was incubated with $160 \mu \mathrm{L}$ of assay buffer (100 mM phosphate buffer, pH 7.3, 102 mM mercaptoethanol and $9 \mathrm{mM} \mathrm{MgCl} 2$ ) and $40 \mu \mathrm{L} 6.25 \mathrm{mM}$ CPRG (chlorophenol red- $\beta$-D-galactopyranoside; Roche, Indianapolis, IN) for $30 \mathrm{~min}$. The enzymatic activity of $\beta$-galactosidase was measured at an absorbance of $570 \mathrm{~nm}$ using a kinetic plate reader (Titertek Multiskan Plus, Flow Laboratories, McLean, VA, USA).

\section{Toxicity assay}

Culture supernatants were collected and measurement of lactate dehydrogenase (LDH) was immediately performed in order to determine cytotoxicity of tested drugs to infected human villous explants. Measurement of LDH released from the cytosol of lysed cell membranes into the supernatant was performed using a commercial diagnostic kit according to manufacturer instructions (LDH Liquiform, LabtestDiagnostica S.A., Lagoa Santa, MG, Brazil). The method used is based on consumption and decrease of absorption of NADH at $340 \mathrm{~nm}$, which was measured in a DU-70 spectrophotometer (Beckman, Brea, CA, USA) for $3 \mathrm{~min}$ at $37^{\circ} \mathrm{C}$. The data were expressed in $\mathrm{U} / \mathrm{mL}$ of enzyme activity. The negative control (uninfected control) was determined by spontaneous LDH released from uninfected villous explants. Maximum LDH released, defined as positive control (total cell lysis), was determined by adding $0.2 \%$ Triton $\mathrm{X}-100$ to explants. In parallel, cytokine and hormone levels were measured in supernatants. The doses of 200 and $1000 \mu \mathrm{g} / \mathrm{mL}$ for azithromycin or pyrimethamine; 150 and $750 \mu \mathrm{g} / \mathrm{mL}$ for sulfadiazine; and 30 to $150 \mu \mathrm{g} / \mathrm{mL}$ for folinic acid were chosen for this latter purpose according to toxicity results. All subsequent experiments were performed using these drug concentrations. Hereafter, PSA concentrations will be denoted based on the concentration of pyrimethamine.

\section{Morphological analysis and immunohistochemistry}

To verify the integrity of villous explants and immunolocalization of parasites, fragments of placental explants were fixed in $10 \%$ buffered formalin, dehydrated in increasing alcohol concentrations, and embedded in paraffin. Sections with $4 \mu \mathrm{m}$ were placed on glass slides and subjected to immunohistochemical analysis [22]. For antigen retrieval, sections were covered with trypsin solution $(0.05 \%$ trypsin and $0.1 \%$ calcium chloride (Sigma-Aldrich Co.) for $30 \mathrm{~min}$ at $37^{\circ} \mathrm{C}$. Explant sections were incubated in $5 \%$ acetic acid at room temperature in order to block endogenous phosphatase activity. Next, sections were incubated overnight at $4^{\circ} \mathrm{C}$ with hyperimmune rabbit anti-T. gondii serum (1:100). Biotinylated goat anti-rabbit IgG (1:600, Sigma-Aldrich Co.) was used as secondary antibody, and the reaction was developed with fast red naphthol (Sigma-Aldrich Co.). Samples were counterstained with Harris's hematoxylin and examined under a light microscope (BX40, Olympus, Tokyo, Japan).

\section{Cytokine and nitrite assays}

Villous explant culture supernatants were analyzed for IL-12, TNF- $\alpha$, IL-10 and TGF- $\beta 1$ concentrations using a sandwich ELISA according to the manufacturer's instructions (R\&D Systems, BD Biosciences, San Diego, CA, USA). Cytokine concentration was determined by extrapolation from a standard curve obtained from known concentrations of the respective recombinant cytokines. Optical density was measured at $450 \mathrm{~nm}$ with a microplate reader (Titertek Multiskan Plus, Flow Laboratories, McLean, VA, USA). The limit of detection of the assays was $7.8 \mathrm{pg} / \mathrm{mL}$ for both TNF- $\alpha$ and IL-12, $62.5 \mathrm{pg} / \mathrm{mL}$ for IL-10, and $31.3 \mathrm{pg} / \mathrm{mL}$ for TGF- $\beta 1$.

Additionally, human cytokines (IL-2, IFN- $\gamma$, IL-6, IL-4 and IL-17A) were quantified using a cytometric bead array $^{\mathrm{TM}}$ (CBA; BD Biosciences) according to the manufacturer's instructions. Samples were analyzed using a $\mathrm{BD}^{\mathrm{TM}}$ flow cytometer (FACSCalibur, BD Biosciences), and data were calculated using specialized $\mathrm{BD}^{\mathrm{TM}}$ Cell Quest and CBA software (BD Biosciences).

Also, supernatants were analyzed by Griess method [25] in order to measure nitrite. Briefly, the supernatants were added in triplicate to 96-well plates and mixed 1:1 
with $1 \%$ sulfanilamide dihydrochloride and $0.1 \%$ naphthylenediamide dihydrochloride in $2.5 \% \mathrm{H} 3 \mathrm{PO}$. Absorbance was read in a plate reader at $570 \mathrm{~nm}$, and concentration was determined with reference to a standard curve of sodium nitrite with concentrations ranging from 5 to $200 \mu \mathrm{mol} / \mathrm{L}$.

\section{Hormone measurement}

Villous explant culture supernatants were also analyzed for concentrations of estradiol, progesterone and HCG $+\beta$ by an electrochemiluminescence immunoassay (ECLIA) intended for use on an automated analyzer Cobas e 411 (Roche Diagnostics GmbH, Mannhein, Germany), according to the manufacturer's instructions. The limit of detection of the assays was $5.0 \mathrm{pg} / \mathrm{mL}$ for estradiol, $0.03 \mathrm{ng} / \mathrm{mL}$ for progesterone and $0.01 \mathrm{mUI} / \mathrm{mL}$ for $\mathrm{HCG}+\beta$.

\section{Measurement of villous explant protein content}

Frozen villous explants were homogenized in radioimmunoprecipitation assay buffer $(50 \mathrm{mM}$ Tris hydrochloride, $150 \mathrm{mM} \mathrm{NaCl}, 1 \%$ (v/v) Triton X-100, 1\% (w/v) sodium deoxycholate and $0.1 \%(\mathrm{w} / \mathrm{v})$ SDS; $\mathrm{pH}$ 7.5) plus a protease inhibitor cocktail (Roche Diagnostics). Homogenate was centrifuged at $15,000 \times g$ for $15 \mathrm{~min}$ at $4^{\circ} \mathrm{C}$. Supernatants were used for protein content measurement using the Bradford method [26]. To normalize explants from different sizes, a ratio between cytokine production $(\mathrm{pg} / \mathrm{mL})$ and its corresponding protein content $(\mathrm{mg} / \mathrm{mL})$ was calculated, and the data were expressed in picograms/milligram $(\mathrm{pg} / \mathrm{mg})$ as previously proposed [21]. Likewise, concentrations of estradiol, progesterone, and $\mathrm{HCG}+\beta$ were normalized according to the corresponding protein content of villous explants, and the data were expressed in $\mathrm{pg} / \mathrm{mg}$, ng/mg and $\mathrm{mIU} / \mathrm{mg}$, respectively.

\section{Statistical analysis}

Data were expressed as mean \pm standard error of mean (SEM), and the differences between groups were determined using ANOVA (with correction for multiple comparisons via the Bonferroni method) or a Student $t$-test, when appropriate (GraphPad Prism Sofware 5.0 GraphPad Software, Inc., San Diego, CA, USA). Differences were considered statistically significant when $\mathrm{P}<0.05$. Six independent experiments were performed in triplicate using 24 placental tissues.

\section{Results}

\section{Antibiotic cytotoxicity is dose dependent}

The degree of cytotoxicity of different doses of antibiotics was evaluated by measuring LDH release in villous explant culture supernatants. T. gondii infection induced greater LDH release and increased cytotoxicity compared to uninfected villous explants (Figure 1A; ANOVA, $\mathrm{P}<0.001$ ). Both drugs at concentrations of 200 or $1000 \mu \mathrm{g} / \mathrm{mL}$ did not cause significant cytotoxicity in villous explants compared to untreated villous explants, regardless of $T$. gondii infection (Figure 1B). However, infected villous explants showed a large increase in cytotoxicity when treated with $5000 \mu \mathrm{g} / \mathrm{mL}$ of PSA compared to untreated villous explants (Figure 1B). Therefore, the concentrations of 200 and $1000 \mu \mathrm{g} / \mathrm{mL}$ were used in further experiments.

\section{Azithromycin and PSA are able to control T. gondii infection in villous explants}

Treatment with either azithromycin or PSA significantly reduced intracellular proliferation of $T$. gondii at both tested concentrations (200 and $1000 \mu \mathrm{g} / \mathrm{mL}$ ) compared to untreated villous explants (Figure 2A; ANOVA, $\mathrm{P}<0.001$ ). In addition, treatment with either drug did not induce morphological alterations in villous explants (Figure 2, B to E). It was observed syncytiotrophoblast covering the chorionic villus, the cytotrophoblast and mesenchymal tissue.

\section{Treatment with azithromycin increases IL-6 production by} T. gondii-infected villous explants

Given the efficiency of both treatments in control of parasitic infection, we next investigated whether antibiotic treatments were able to alter cytokine production by villous explants. Analyses of cytokine levels showed that azithromycin and PSA treatments at 200 and $1000 \mu \mathrm{g} / \mathrm{mL}$ did not alter TNF- $\alpha$, IL-17A or TGF- $\beta 1$ production by infected villous explants compared to untreated/infected controls (NT) (Figure 3, A to C; ANOVA, $\mathrm{P}<0.001$ ). However, a decrease in the $T$. gondii proliferation in villous explants treated with either drug was observed.

T. gondii-infected villous explants produced lower levels of IL-10 and a significant increase of IL-12 after treatment with PSA at both concentrations. Decreased IL-10 levels and increased IL-12 levels matched with reduced parasite load (Figure 3, D and E; ANOVA, P < 0.001).

When infected villous explants were treated with azithromycin at $200 \mu \mathrm{g} / \mathrm{mL}$, an increase in IL-6 levels was observed relative to untreated/infected controls (Figure 3F; ANOVA, $\mathrm{P}<0.001$ ). IL-2, IFN- $\gamma$ and IL-4 levels were not detectable in any culture supernatants.

\section{T. gondii infection increases estradiol, progesterone and HCG $+\beta$ production in villous explants and antibiotic treatment downmodulates these hormones}

In order to better understand the action of antibiotics on the physiology of villous explants, we conducted a second set of experiments to determine whether $T$. gondii infection is able to alter the hormonal production profile during treatment with azithromycin or PSA. Increased secretion of estradiol, progesterone and HCG $+\beta$ in supernatants of $T$. gondii-infected villous explants was observed compared to their respective controls (Figure 4, A to C; ANOVA, $\mathrm{P}<0.001)$. However, when infected villous explants were 

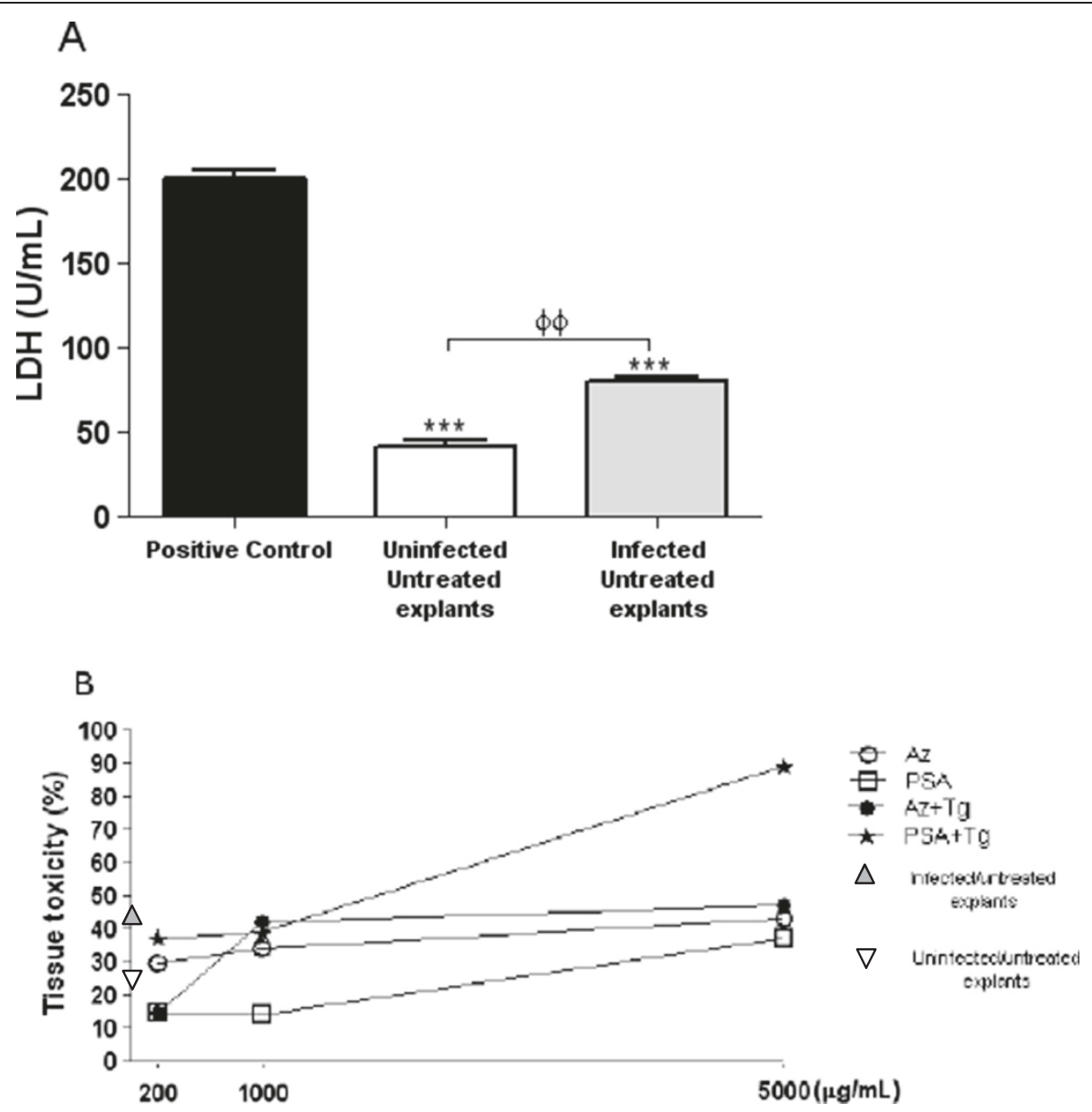

Figure 1 Analysis of cytotoxicity in human villous explants. Villous explant supernatants were collected and lactate dehydrogenase (LDH) activity was measured using the LDH Liquiform kit. The positive control was established by adding $0.2 \%$ Triton X-100 (A). Human villous explants were infected or not by T. gondii and treated with increasing concentrations of azithromycin or PSA for $24 \mathrm{~h}$ and the percentage of tissue toxicity was analyzed (B). Data are expressed as mean \pm SEM. Significant differences were analyzed by ANOVA and Bonferroni multiple comparison post hoc test. ${ }^{* * *} \mathrm{P}<0.001$ in relation to the positive control; ${ }^{\Phi \Phi} \mathrm{P}<0.01$ between uninfected and infected villous explants.

treated with azithromycin, a reduction in the secretion of estradiol and $\mathrm{HCG}+\beta$ was concomitant with a decrease in parasite load (Figure 4, D to F; ANOVA, P < 0.001). PSA treatment of $T$. gondii-infected villous explants induced a significant decrease in estradiol and progesterone compared to infected/untreated controls (Figure 4, D to F, ANOVA, $\mathrm{P}<0.001$ ). Simultaneously, there was a reduction in parasite load. In contrast, the effects of PSA on HCG $+\beta$ secretion showed a significant reduction in the hormone secretion with respect of infected untreated explants, although no difference was observed between the two concentrations tested. However, PSA treatment reduced the parasite load.

\section{Pretreatment of $T$. gondii tachyzoites with azithromycin} or PSA reduces infection in villous explants

The direct effect of azithromycin or PSA on parasites was subsequently investigated. $T$. gondii $2 \mathrm{~F} 1$ tachyzoites were pretreated with different concentrations (50, 100, 200 and
$1000 \mu \mathrm{g} / \mathrm{mL}$ ) of the two drugs (Figure 5). Azithromycin and PSA pretreatment at all concentrations reduced proliferation of $T$. gondii compared to controls, but a dosedependent relationship was only observed for azithromycin (Figure 5; ANOVA, $\mathrm{P}<0.05$ ), suggesting a direct action on the parasite.

\section{Production of nitrite}

No production of nitrite was detected in supernatants from villous explants in all experiments, suggesting that nitrogen monoxide (NO) is not involved in the reduction of parasite load in this experimental design.

\section{Discussion}

The conventional treatment for toxoplasmosis in pregnant women is based on administration of spiramycin or a combination of pyrimethamine, sulfadiazine and folinic acid (PSA) in cases of fetal infection [23]. Pyrimethamine 

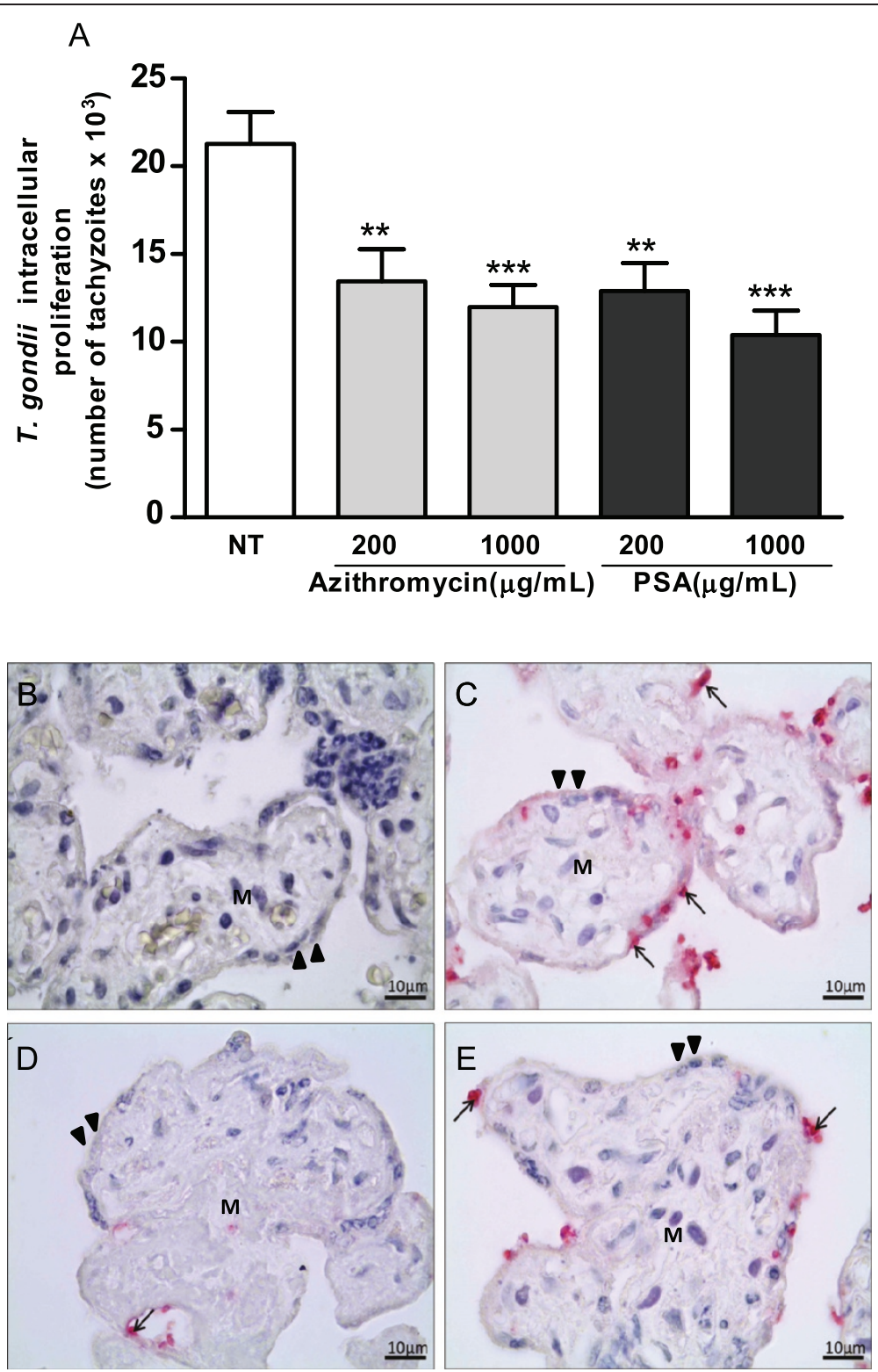

Figure 2 T. gondii intracellular proliferation in villous explants treated with azithromycin or PSA. Villous explants were infected and treated for $72 \mathrm{~h}$ with azithromycin or PSA at different concentrations. T. gondii proliferation was measured by a colorimetric $\beta$-galactosidase assay (A). The bars represent mean \pm SEM. Data were analyzed by ANOVA and Bonferroni multiple comparison post hoc test. Significant differences in comparison with untreated villous explants are labeled $\left({ }^{* * *} \mathrm{P}<0.001 ;{ }^{* *} \mathrm{P}<0.01\right)$. Representative photomicrographs of human villous explants infected or not with T. gondii: uninfected human villous explants as a negative control (B), arrows indicate tachyzoites in untreated human villous explants (C), azithromycin-treated (D) and PSA-treated (E) human villous explants. Arrowheads indicate syncytiotrophoblast layers, and $\mathbf{M}$ indicates mesenchymal tissue. Counterstaining with Mayer's hematoxylin was performed. Scale bar: $10 \mu \mathrm{m}$.

is a potentially teratogenic antibiotic and should not be used during the first trimester of pregnancy [8]. Thus, it is essential to develop new drugs, as well as new therapeutic approaches, for the treatment of congenital toxoplasmosis. Here, we showed that azithromycin is as effective as PSA treatment of human placental villous infected with T. gondii.

Drug dosages used (200 and $1000 \mu \mathrm{g} / \mathrm{mL}$ for both azithromycin and PSA) were selected on the basis of lowest cytotoxicity, which was indirectly assessed by release of LDH. Both azithromycin and PSA were able to reduce $T$. gondii proliferation at both tested concentrations (200 and $1000 \mu \mathrm{g} / \mathrm{mL})$. These data are in agreement with our previous studies, where azithromycin treatment was able to control T. gondii infection in human trophoblast BeWo cells [19]. Additionally, azithromycin also showed a protective role against the ME49 strain of $T$. gondii in rodent $C$. callosus, reducing vertical transmission 


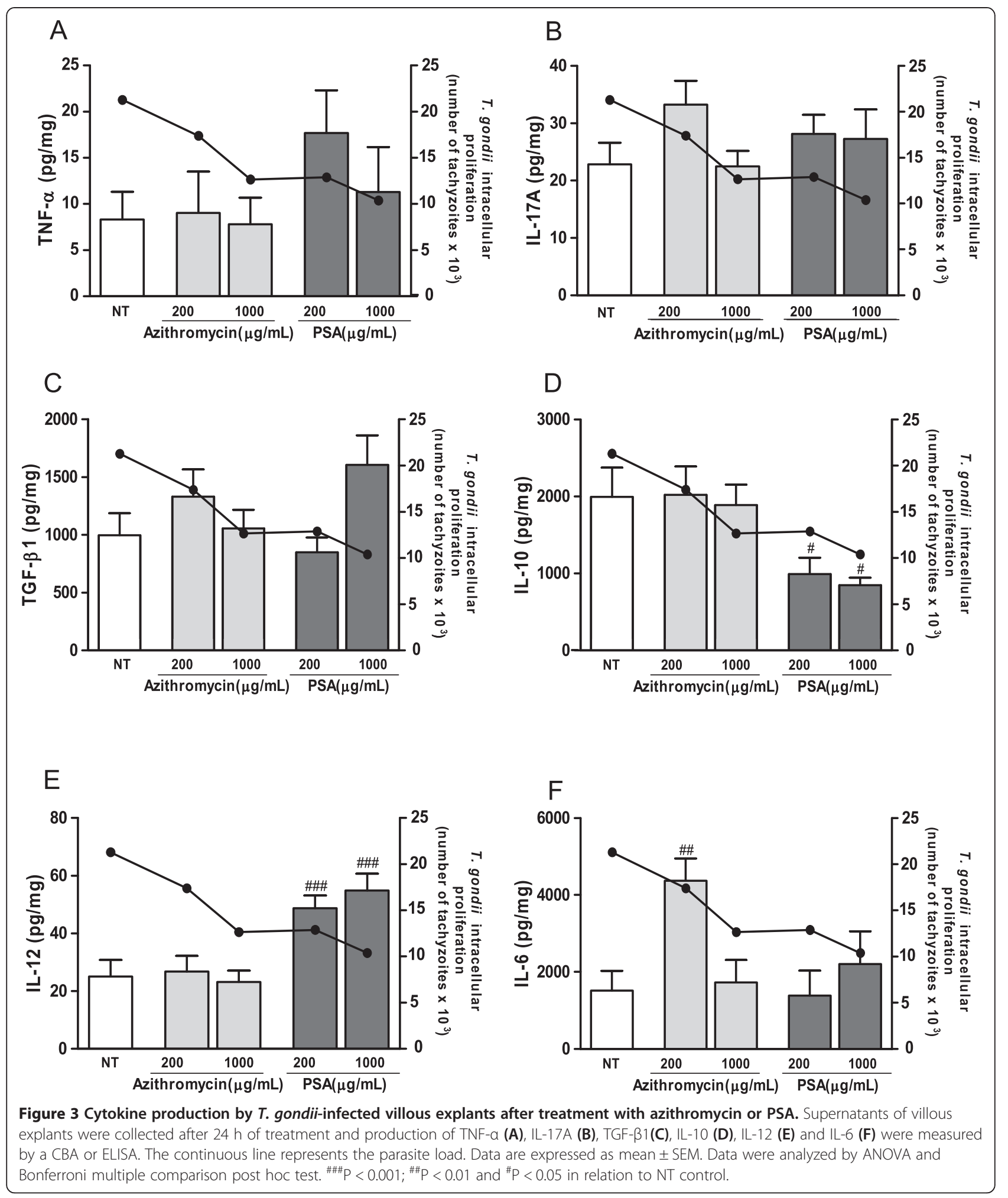

of the parasite [18]. In the latter study, the number of tachyzoites and bradyzoites of T. gondii decreased significantly compared to untreated or PSA-treated C. callosus, demonstrating the effectiveness of azithromycin treatment [18]. In another study, azithromycin also reduced $T$. gondii infection in maternal brains and fetal eyes in a C. callosus model of congenital toxoplasmosis [27]. 


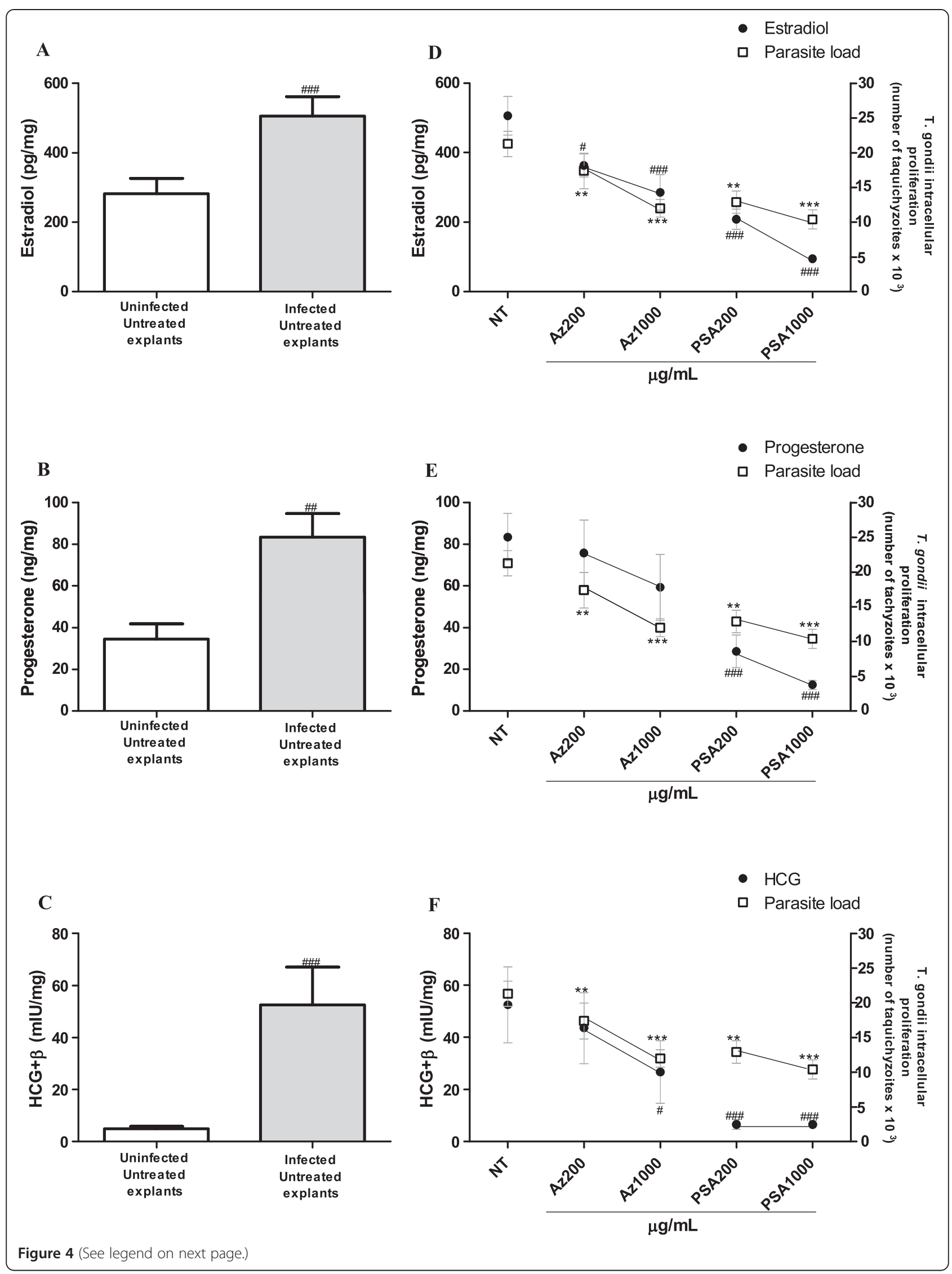


(See figure on previous page.)

Figure 4 Detection of sex hormones and parasite load in uninfected and $T$. gondii-infected villous explants, treated or not with azithromycin or PSA. Estradiol (A), progesterone (B) and HCG $+\beta$ (C) were measured by an electrochemiluminescence immunoassay of uninfected or T. gondii-infected villous explants. Levels of (D) estradiol, (E) progesterone, and (F) HCG + $\beta$ in azithromycin- or PSA-treated or untreated villous explants were analyzed in relation to parasite load. Data were analyzed by ANOVA and Bonferroni multiple comparison post hoc test. Comparisons are represented by symbols: parasitism, ${ }^{* * * *} \mathrm{P}<0.001,{ }^{* * *} \mathrm{P}<0.01,{ }^{*} \mathrm{P}<0.05$ and sexual hormones, ${ }^{\# \# \#} \mathrm{P}<0.001,{ }^{\# \#} \mathrm{P}<0.01,{ }^{\#} \mathrm{P}<0.05$ in relation to NT control.

Azithromycin can be administered safely during the three trimesters of pregnancy and does not have teratogenic effects $[17,28,29]$. A recent study demonstrated hopeful results in the treatment of congenital toxoplasmosis by maternal oral administration of azithromycin in addition to sulfadoxine, pyrimethamine and acetylspiramycin in a case of severe symptomatic fetal toxoplasmosis [7].

We observed that $T$. gondii infection reduced significantly the IL- 6 production when compared to untreated and uninfected villous explants, while azithromycin treatment in uninfected villous explants did not alter the IL-6 production (data not shown). However, the level of this cytokine was restored when the infected villous explants were treated with azithromycin. In addition, azithromycin treatment did not alter the production of TNF- $\alpha$, IL-17A, TGF- $\beta 1$, IL-10 or IL-12 cytokines. Macrolide antibiotics not only have antibacterial activity, but also antiinflammatory activity and immunomodulatory effects [30]. Previous studies from our group have shown that azithromycin treatment induces an anti-inflammatory response in trophoblastic cells infected by $T$. gondii [19]. However, in the present work, we observed changes only in IL-6 levels, without any variation in the cytokine profile when $T$. gondii-infected villous explants were treated with azithromycin. Some studies have demonstrated the suppressor effect of azithromycin in the IL-6 production. Azithromycin reduced significantly the IL-6 release in macrophages [31-33] and in the serum of pregnant women infected by Chlamydia trachomatis [15], evidencing the anti-inflammatory role of this drug in many experimental models. Some undesirable effects of the anti-inflammatory activity of macrolides include human gingival cells when treated with azithromycin or erythromycin showed no inhibition of matrix metalloproteinase 9 or CXCL8 [34]. Furthermore, clarithromycin or azithromycin has not a beneficial effect on CXCL8 release by human whole blood cells and alveolar macrophages [35]. These data suggest that the antiinflammatory action of azithromycin is dependent on cell type and microenvironment. Furthermore, there is evidence that IL-6 plays an important role in resistance to T. gondii infection [36,37]. In addition, this cytokine participates in migration and invasion of trophoblasts into the endometrium [38], inducing release of $\mathrm{HCG}+\beta$ in BeWo cells [39] and other models of human trophoblast [40]. On the other hand, increased levels of IL-6 are involved in several aspects of pathogenesis during pre-eclampsia [41].

Conversely, PSA treatment reduced production of IL-10 and increased IL-12 levels. These results suggest a

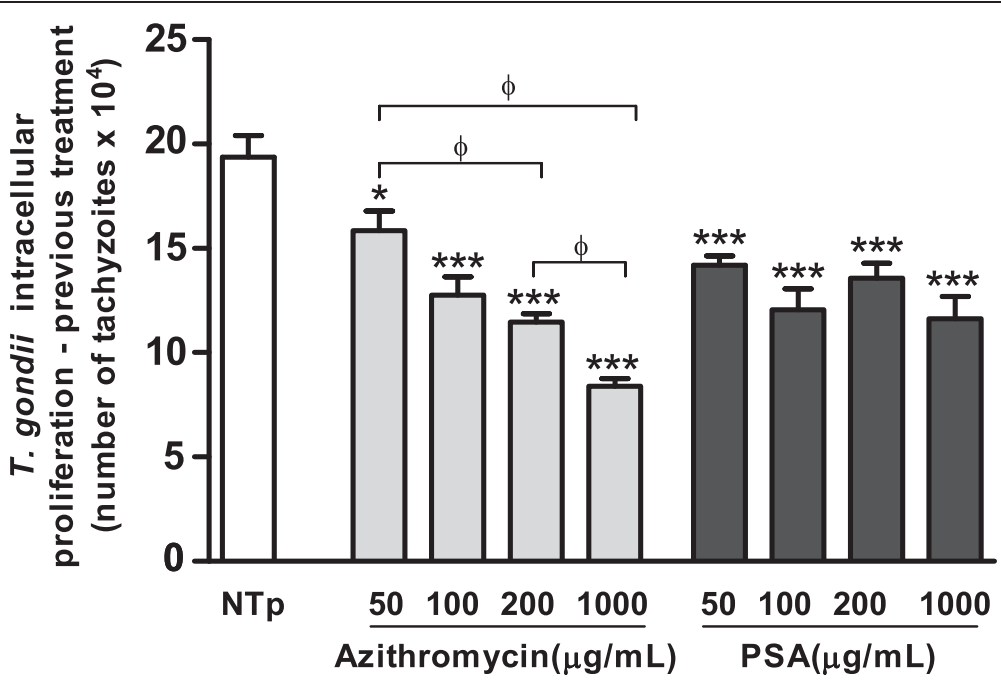

Figure 5 T. gondii intracellular proliferation in villous explants infected with pretreated (azithromycin or PSA) parasites. Proliferation was measured by a colorimetric $\beta$-galactosidase assay. The bars represent mean \pm SEM. Data were analyzed by ANOVA and Bonferroni multiple comparison post hoc test. ${ }^{* * *} \mathrm{P}<0.001,{ }^{* * *} \mathrm{P}<0.01$ and ${ }^{*} \mathrm{P}<0.05$ in relation to NTp control. ${ }^{\oplus}$ Comparison between villous explants treated with different concentrations of azithromycin $(P<0.05)$. 
potential pro-inflammatory effect of PSA treatment, although we did not detect production of IFN- $\gamma$. During pregnancy, induction of a type- 2 immune response has no effect on the pregnancy; however, induction of a type- 1 response by parasitic infection can lead to abortion via alteration of the cytokine profile in the fetal-maternal interface [42]. In murine models of congenital toxoplasmosis, an imbalance in cytokine production and increased inflammatory responses are associated with increased apoptosis and necrosis at the site of implantation, leading to embryonic loss [43].

In the present study, we showed that $T$. gondii infection increased production of estradiol, progesterone and $\mathrm{HCG}+\beta$ in villous explants. Azithromycin did not alter the estradiol, progesterone or $\mathrm{HCG}+\beta$ release in uninfected villous, while PSA increased only estradiol and progesterone production at the same condition (data not shown). Thus, these findings demonstrated that azithromycin did not alter the normal hormone profile. In addition, treatment with azithromycin or PSA was able to reduce secretion of these hormones when compared to infected but untreated tissues. It has been described that for some parasitic diseases, as leishmaniasis, toxoplasmosis and malaria, increased levels of progesterone and glucocorticoid are associated with decreased resistance in murine experimental models [44-46]. In mice, increased morbidity during toxoplasmosis has been associated with estradiol levels, while gonadectomy reduces pathogenesis of toxoplasmosis [3]. A high concentration of progesterone has also been shown to increase susceptibility to $T$. gondii infection during pregnancy by suppressing production of IL-12 and IFN- $\gamma$ [47] and, therefore, suppressing a type-1 response utilizing the progesterone and glucocorticoid receptors [48]. In the present work, increased production of hormones by $T$. gondii-infected villous explants may be a result of an evasion strategy orchestrated by the parasite. Recent works have demonstrated that hormones are deeply involved in the regulation of parasite load both modulating the host immune-endocrine network and parasite proliferation [49]. Thus we hypothesized an indirect action of the drug treatment inducing lower parasite load that in turn decreased hormones secretion. We showed that $T$. gondii-infected villous explants, treated or untreated, failed to induce NO production. A previous study has demonstrated that human villous explants as well BeWo trophoblast cells infected with $T$. gondii were unable to release NO $[21,50]$. In our experimental design, reduction of parasite load after antibiotic treatment was likely not related to NO.

Our results also showed that pretreatment of parasites with azithromycin or PSA was able to reduce proliferation of $T$. gondii in villous explants. Azithromycin targets the $70 \mathrm{~S}$ ribosomal subunit of susceptible microorganisms, such as $P$. falciparum and $P$. vivax, interfering with protein synthesis of the parasites [17]. Additionally, parasites from the Apicomplexa phylum have a plastid called apicoplast, which is a target site for binding of diverse drugs with antiparasitic activity $[51,52]$. On the other hand, blocking the activities of the plastid does not typically result in immediate death of parasites, including T. gondii. In contrast, the parasiticidal activity of plastid inhibitors is only detected when tachyzoites attempt to establish infection within a new host cell $[17,53]$.

\section{Conclusion}

The present study demonstrated that azithromycin is able to control $T$. gondii infection in human villous explants from the third trimester of pregnancy, providing evidence that it may be an effective alternative drug for treatment of congenital toxoplasmosis by reducing the proliferation rate of $T$. gondii. Further clinical studies need to be conducted to validate the present findings and to enable new therapeutic approaches for the treatment of $T$. gondii infection at the fetal-maternal interface.

\section{Ethical approval}

The present study had the approval of the Human Research Ethics Committee of the Universidade Federal de Uberlândia, Brazil. Informed consent from all patients was obtained.

\section{Abbreviations}

PSA: Pyrimethamine, sulfadiazine and folinic acid; RPMI 1640: Roswell Park Memorial Institute medium; FBS: Fetal bovine serum; NT: Infected/untreated villous explants; NTp: Untreated parasites; LDH: Lactate dehydrogenase; CBA: Cytometric bead array; ECLIA: Electrochemiluminescence immunoassay; NO: nitrogen monoxide.

\section{Competing interests}

All authors declare that they have no competing interests.

\section{Authors' contributions}

All authors contributed in one or more of the following ways: LSCF, BFB and EAVF wrote the manuscript. DAOS and JRM revised the manuscript. LSCF, $A O G$, and MCS performed the collection of placental tissues and experiments involving infection and treatment. LSCF performed all hormone measurements. LSCF and CMOSA performed all toxicity assays. LSCF and MBA performed all ELISAs and CBA experiments. EAVF, NMS, DAOS and JRM contributed to reagent preparation and performed the quantitative analysis. EAVF, OAMF, NMS, DAOS and JRM provided suggestions and valuable discussion throughout the study. We hereby declare that all authors have read and approved the manuscript.

\section{Acknowledgements}

This work was supported by Brazilian Research Funding Agencies: Conselho Nacional de Desenvolvimento Científico e Tecnológico (CNPq), Fundação de Amparo à Pesquisa do Estado de Minas Gerais (FAPEMIG) and Coordenadoria de Aperfeiçoamento de Pessoal do Ensino Superior (CAPES). The authors thank the Laboratory of Clinical Analyses of the Universidade Federal de Uberlândia for the technical support.

\section{Author details}

'Laboratory of Immunophysiology of Reproduction, Institute of Biomedical Sciences, Universidade Federal de Uberlândia, Av. Pará 1720, Uberlândia, MG 38400-902, Brazil. 'Laboratory of Immunopathology, Institute of Biomedical Sciences, Universidade Federal de Uberlândia, Av. Pará 1720, Uberlândia, MG 
38400-902, Brazil. ${ }^{3}$ Laboratory of Chagas Disease, René Rachou Research Center, Fundação Oswaldo Cruz, Belo Horizonte, MG 30190-002, Brazil. ${ }^{4}$ Department of Gynecology and Obstetrics, Faculty of Medicine, Universidade Federal de Uberlândia, Uberlândia, MG, Brazil.

Received: 19 December 2013 Accepted: 14 May 2014 Published: 19 May 2014

\section{References}

1. Faucher B, Garcia-Meric P, Franck J, Minodier P, Francois P, Gonnet S, L'Ollivier $C$, Piarroux R: Long-term ocular outcome in congenital toxoplasmosis: a prospective cohort of treated children. J Infect 2012, 64:104-109.

2. Oreshkova T, Dimitrov R, Mourdjeva M: A cross-talk of decidual stromal cells, trophoblast, and immune cells: a prerequisite for the success of pregnancy. Am J Reprod Immunol 2012, 68:366-373.

3. Robinson DP, Klein SL: Pregnancy and pregnancy-associated hormones alter immune responses and disease pathogenesis. Horm Behav 2012, 62:263-271.

4. Wallon M, Peyron F, Cornu C, Vinault S, Abrahamowicz M, Kopp CB, Binquet $C$ : Congenital toxoplasma infection: monthly prenatal screening decreases transmission rate and improves clinical outcome at age 3 years. Clin Infect Dis 2013, 56:1223-1231.

5. Bar-Oz B, Diav-Citrin O, Shechtman S, Tellem R, Arnon J, Francetic I, Berkovitch M, Ornoy A: Pregnancy outcome after gestational exposure to the new macrolides: a prospective multi-center observational study. Eur $\lrcorner$ Obstet Gynecol Reprod Biol 2008, 141:31-34.

6. Elsheikha HM: Congenital toxoplasmosis: priorities for further health promotion action. Public Health 2008, 122:335-353.

7. Tamaru S, Kikuchi A, Takagi K, Wakamatsu M, Horikoshi T, Ogiso Y: Fetal therapy of severe symptomatic toxoplasmosis using azithromycin. J Obstet Gynaecol Res 2011, 37:953-957.

8. Montoya JG, Remington JS: Management of Toxoplasma gondii infection during pregnancy. Clin Infect Dis 2008, 47:554-566.

9. Hernandez-Diaz S, Werler MM, Walker AM, Mitchell AA: Neural tube defects in relation to use of folic acid antagonists during pregnancy. Am J Epidemiol 2001, 153:961-968.

10. Vijayalaxmi KK, Vishalakshi M: Evaluation of the genotoxic effects of pyrimethamine, an antimalarial drug, in the in vivo mouse. Teratog Carcinog Mutagen 2000, 20:65-71.

11. Degerli K, Kilimcioglu AA, Kurt O, Tamay AT, Ozbilgin A: Efficacy of azithromycin in a murine toxoplasmosis model, employing a Toxoplasma gondii strain from Turkey. Acta Trop 2003, 88:45-50.

12. Ramsey PS, Vaules MB, Vasdev GM, Andrews WW, Ramin KD: Maternal and transplacental pharmacokinetics of azithromycin. Am J Obstet Gynecol 2003, 188:714-718.

13. Salman S, Rogerson SJ, Kose K, Griffin S, Gomorai S, Baiwog F, Winmai J, Kandai J, Karunajeewa HA, O'Halloran SJ, Siba P, llett KF, Mueller I, Davis TME: Pharmacokinetic properties of azithromycin in pregnancy. Antimicrob Agents Chemother 2010, 54:360-366.

14. Pitsouni E, lavazzo C, Athanasiou S, Falagas ME: Single-dose azithromycin versus erythromycin or amoxicillin for Chlamydia trachomatis infection during pregnancy: a meta-analysis of randomised controlled trials. Int J Antimicrob Agents 2007, 30:213-221.

15. Srivastava P, Bhengraj AR, Jha HC, Vardhan $H$, Jha R, Singh LC, Salhan $S$, Mittal A: Differing effects of azithromycin and doxycycline on cytokines in cells from Chlamydia trachomatis-infected women. DNA Cell Biol 2012, 31:392-401.

16. Briggs GG, Freeman RK, Yaffe SJ: Drugs in pregnancy and lactation. 9th edition. Philadelphia: Williams \& Wilkins; 2011.

17. Chico RM, Chandramohan D: Azithromycin plus chloroquine: combination therapy for protection against malaria and sexually transmitted infections in pregnancy. Expert Opin Drug Metab Toxicol 2011, 7:1153-1167.

18. Costa IN, Angeloni MB, Santana $L A$, Barbosa BF, Silva MC, Rodrigues $A A$, Rostkowsa C, Magalhaes PM, Pena JD, Silva DA, Mineo JR, Ferro EA: Azithromycin inhibits vertical transmission of Toxoplasma gondii in Calomys callosus (Rodentia: Cricetidae). Placenta 2009, 30:884-890.

19. Franco PS, Gomes AO, Barbosa BF, Angeloni MB, Silva NM, Teixeira-Carvalho A, Martins-Filho OA, Silva DA, Mineo JR, Ferro EA: Azithromycin and spiramycin induce anti-inflammatory response in human trophoblastic (BeWo) cells infected by Toxoplasma gondii but are able to control infection. Placenta 2011, 32:838-844.
20. Rubin BK: Immunomodulatory properties of macrolides: overview and historical perspective. Am J Med 2004, 117(Suppl 9A):2S-4S.

21. Gomes AO, Silva DAO, Silva NM, Barbosa BF, Franco PS, Angeloni MB, Fermino ML, Roque-Barreira MC, Bechi N, Paulesu LR, Dos Santos MC, Mineo JR, Ferro EA: Effect of macrophage migration inhibitory factor (MIF) in human placental explants infected with Toxoplasma gondii depends on gestational age. Am J Pathol 2011, 178:2792-2801.

22. Ferro EA, Silva DA, Bevilacqua E, Mineo JR: Effect of Toxoplasma gondii infection kinetics on trophoblast cell population in Calomys callosus, a model of congenital toxoplasmosis. Infect Immun 2002, 70:7089-7094.

23. Remington JS, McLeod R, Thulliez P, Desmonts G: Infectious diseases of the fetus and newborn infant. 6th edition. Philadelphia: Saunders; 2006.

24. Teo CF, Zhou XW, Bogyo M, Carruthers VB: Cysteine protease inhibitors block Toxoplasma gondii microneme secretion and cell invasion. Antimicrob Agents Chemother 2007, 51:679-688.

25. Green LC, Wagner DA, Glogowski J, Skipper PL, Wishnok JS, Tannenbaum SR: Analysis of nitrate, nitrite, and [15 N] nitrate in biological fluids. Anal Biochem 1982, 126:131-138.

26. Bradford MM: A rapid and sensitive method for the quantitation of microgram quantities of protein utilizing the principle of protein-dye binding. Anal Biochem 1976, 72:248-254.

27. Lopes CD, Silva NM, Ferro EA, Sousa RA, Firminot ML, Bernardes ES, Roque-Barreira MC, Pena JD: Azithromycin reduces ocular infection during congenital transmission of toxoplasmosis in the Calomys callosus model. J Parasitol 2009, 95:1005-1010.

28. Chico RM, Pittrof R, Greenwood B, Chandramohan D: Azithromycin-chloroquine and the intermittent preventive treatment of malaria in pregnancy. Malar J 2008, 7:255.

29. Pereira MR, Henrich PP, Sidhu AB, Johnson D, Hardink J, Van Deusen J, Lin J, Gore K, O'Brien C, Wele M, Djimde A, Chandra R, Fidock DA, In vivo and in vitro antimalarial properties of azithromycin-chloroquine combinations that include the resistance reversal agent amlodipine. Antimicrob Agents Chemother 2011, 55:3115-3124.

30. Kanoh S, Rubin BK: Mechanisms of action and clinical application of macrolides as immunomodulatory medications. Clin Microbiol Rev 2010, 23:590-615.

31. Choi EY, Jin JY, Choi JI, Choi IS, Kim SJ: Effect of azithromycin on Prevotella intermedia lipopolysaccharide-induced production of interleukin- 6 in murine macrophages. Eur J Pharmacol 2014, 729:10-16.

32. Cai M, Bonella F, Dai H, Sarria R, Guzman J, Costabel U: Macrolides inhibit cytokine production by alveolar macrophages in bronchiolitis obliterans organizing pneumonia. Immunobiology 2013, 218:930-937.

33. Banjanac M, Munic Kos V, Nujic K, Vrancic M, Belamaric D, Crnkovic S, Hlevnjak M, Erakovic Haber V: Anti-inflammatory mechanism of action of azithromycin in LPS-stimulated J774A.1 cells. Pharmacol Res 2012, 66:357-362.

34. Kamemoto A, Ara T, Hattori T, Fujinami Y, Imamura Y, Wang PL: Macrolide antibiotics like azithromycin increase lipopolysaccharide-induced IL-8 production by human gingival fibroblasts. Eur J Med Res 2009, 14:309-314.

35. Kurdowska A, Noble JM, Griffith DE: The effect of azithromycin and clarithromycin on ex vivo interleukin-8 (IL-8) release from whole blood and IL-8 production by human alveolar macrophages. J Antimicrob Chemother 2001, 47:867-870.

36. Castro AS, Alves CM, Angeloni MB, Gomes AO, Barbosa BF, Franco PS, Silva DA, Martins-Filho OA, Mineo JR, Mineo TW, Ferro EA: Trophoblast cells are able to regulate monocyte activity to control Toxoplasma gondii infection. Placenta 2013, 34:240-247.

37. Silver JS, Stumhofer JS, Passos S, Ernst M, Hunter CA: IL-6 mediates the susceptibility of glycoprotein 130 hypermorphs to Toxoplasma gondii. $J$ Immunol 2011, 187:350-360.

38. Meisser A, Cameo P, Islami D, Campana A, Bischof P: Effects of interleukin-6 (IL-6) on cytotrophoblastic cells. Mol Hum Reprod 1999, 5:1055-1058.

39. Tsukihara S, Harada T, Deura I, Mitsunari M, Yoshida S, Iwabe T, Terakawa N: Interleukin-1 beta-induced expression of IL-6 and production of human chorionic gonadotropin in human trophoblast cells via nuclear factor-kappaB activation. Am J Reprod Immunol 2004, 52:218-223.

40. Li Y, Matsuzaki N, Masuhiro K, Kameda T, Taniguchi T, Saji F, Yone K, Tanizawa O: Trophoblast-derived tumor necrosis factor-alpha induces release of human chorionic gonadotropin using interleukin-6 (IL-6) and IL-6-receptor-dependent system in the normal human trophoblasts. J Clin Endocrinol Metab 1992, 74:184-191. 
41. Uchide N, Ohyama K, Bessho T, Takeichi M, Toyoda H: Possible roles of proinflammatory and chemoattractive cytokines produced by human fetal membrane cells in the pathology of adverse pregnancy outcomes associated with influenza virus infection. Mediators Inflamm 2012, 2012:270670.

42. Agrawal V, Hirsch E: Intrauterine infection and preterm labor. Semin Fetal Neonatal Med 2012, 17:12-19.

43. Coutinho LB, Gomes AO, Araujo EC, Barenco PV, Santos IL, Caixeta DR, Silva DA, Cunha-Junior JP, Ferro EA, Silva NM: The impaired pregnancy outcome in murine congenital toxoplasmosis is associated with a pro-inflammatory immune response, but not correlated with decidual inducible nitric oxide synthase expression. Int J Parasitol 2012, 42:341-352.

44. Krishnan L, Guilbert L, Russell AS, Wegmann TG, Mosmann TR, Belosevic M: Pregnancy impairs resistance of C57BL/6 mice to Leishmania major infection and causes decreased antigen-specific IFN-gamma response and increased production of T helper 2 cytokines. J Immunol 1996, 156:644-652.

45. Luft BJ, Remington JS: Effect of pregnancy on resistance to Listeria monocytogenes and Toxoplasma gondii infections in mice. Infect Immun 1982, 38:1164-1171.

46. Menendez C: Malaria during pregnancy: a priority area of malaria research and control. Parasitol Today 1995, 11:178-183.

47. Jones LA, Kreem S, Shweash M, Paul A, Alexander J, Roberts CW: Differential modulation of TLR3- and TLR4-mediated dendritic cell maturation and function by progesterone. J Immunol 2010, 185:4525-4534.

48. Jones LA, Anthony JP, Henriquez FL, Lyons RE, Nickdel MB, Carter KC, Alexander J, Roberts CW: Toll-like receptor-4-mediated macrophage activation is differentially regulated by progesterone via the glucocorticoid and progesterone receptors. Immunology 2008, 125:59-69.

49. Escobedo G, Roberts CW, Carrero JC, Morales-Montor J: Parasite regulation by host hormones: an old mechanism of host exploitation? Trends Parasitol 2005, 21:588-593.

50. Pfaff AW, Villard O, Klein JP, Mousli M, Candolfi E: Regulation of Toxoplasma gondii multiplication in BeWo trophoblast cells: cross-regulation of nitric oxide production and polyamine biosynthesis. Int J Parasitol 2005, 35:1569-1576

51. Fichera ME, Roos DS: A plastid organelle as a drug target in apicomplexan parasites. Nature 1997, 390:407-409.

52. Muller J, Hemphill A: Drug target identification in intracellular and extracellular protozoan parasites. Curr Top Med Chem 2011, 11:2029-2038.

53. McFadden Gl, Roos DS: Apicomplexan plastids as drug targets. Trends Microbiol 1999, 7:328-333.

doi:10.1186/1479-5876-12-132

Cite this article as: Castro-Filice et al:: Azithromycin is able to control Toxoplasma gondii infection in human villous explants. Journal of Translational Medicine 2014 12:132.

\section{Submit your next manuscript to BioMed Central and take full advantage of:}

- Convenient online submission

- Thorough peer review

- No space constraints or color figure charges

- Immediate publication on acceptance

- Inclusion in PubMed, CAS, Scopus and Google Scholar

- Research which is freely available for redistribution

Submit your manuscript at www.biomedcentral.com/submit
( Biomed Central 